\title{
The impact of systemic lupus erythematosus on female sexual dysfunction in china
}

\author{
Ying-Hua Pan ${ }^{1 *}$, Zhan-Qin Shao ${ }^{1}$, Yu-Hua Deng ${ }^{1}$, Lei He ${ }^{2}$, Heng-Ying Fang ${ }^{3}$ and Dan-Chun $\mathrm{Wu}^{2 *}$ \\ ${ }^{1}$ Department of Rheumatology, The Third Affiliated Hospital of Sun Yat-sen University, Guangzhou 510600, China \\ ${ }^{2}$ Department of General medicine, The Third Affiliated Hospital of Sun Yat-sen University, Guangzhou 510600, China \\ ${ }^{3}$ Department of Nursing, The Third Affiliated Hospital of Sun Yat-sen University, Guangzhou 510600, China
}

\begin{abstract}
Purpose: Systemic lupus erythematosus (SLE) is a chronic autoimmune disease involving multiple organs. The clinical treatment of SLE is mainly to control disease activity and improve the life quality of patients. Sexual life is an important part of quality of life, but the current status of sexual dysfunction in women with SLE in China and the related influencing factors are unclear.

Methods: From April 2008 to May 2019, a total of 214 women with SLE were recruited from a questionnaire survey in the Third Affiliated Hospital of Sun Yat-sen University. The questionnaire consisted of three parts: general information, the Female Sexual Function Index Scale (FSFI), the Concise Quality of Life Evaluation Scale (SF-36) and Hospital Anxiety and Depression Scale (HADS). The association of SLE and female sexual dysfunction (FSD) was determined by statistical analyses.

Results: A total of 214 questionnaires were distributed, and 204 were returned. Among them, 141 women with SLE (69.1\%) also had sexual dysfunction. The total FSFI score was $21.4 \pm 8.6$. The scores of sexual desire, arousal, vaginal lubrication, orgasm, satisfaction, and intercourse pain dimensions were $2.8 \pm 1.2,3.12 \pm 1.6$, $4.0 \pm 1.9,3.4 \pm 1.7,3.9 \pm 1.4$, and $4.1 \pm 1.9$, respectively. According to correlation analyses, age, factors in SF-36 and HADS forms, and the clinical symptoms including organs involvement, facial rash, arthralgia and Raynaud's phenomenon were the main factors affecting the sexual function of female SLE patients, whereas the status of marriage, fertility, work and dwelling, as well as education, the payment method, yearly income were not involved in the sexual dysfunction. However, effective treatments may contribute to the improvement of sexual function of female SLE patients. Multivariate linear regression analyses showed that the sexual function of female patients with SLE was affected by many factors that contribute to the disease progression.
\end{abstract}

Conclusion: The incidence of FSD in patients with SLE in China is high. The main influencing factors were closely associated the progression of the disease. Strengthening control of these factors may help to improve the quality of sexual life of female patients with SLE.

\section{Introduction}

Systemic lupus erythematosus (SLE) is a chronic autoimmune disease involving multiple organs and a variety of autoantibodies in the body. SLE mainly occurs in young female patients, and the peak onset age is 20 to 40 years old [1]. T lymphocytes and B lymphocytes are highly activated and dysfunctional in patients with SLE, with production of an overabundance of autoantibodies leading to the damage of multiple organs [2]. The disease is known for frequent relapses, negative side effects of drug treatment, and poor clinical prognosis. SLE is accompanied by a variety of clinical manifestations, primarily systemic tissue damage such as vasculitis, Raynaud phenomenon, and endothelial dysfunction [3]. A variety of prominent psychological problems such as anxiety, depression, and sadness accompany physical changes and impaired organ function, seriously affecting patients' rehabilitation and life quality [4].

Sexual life is a basic part of quality of life. For female patients with chronic diseases, the quality of sexual life and sexual function affect the patient physically, psychologically, and socially [5]. Patients with a chronic disease are prone to sexual dysfunction after onset and during treatment. Sexual dysfunction refers to disorders that occur in one or more aspects of the female sexual response cycle, mainly sexual desire disorders, sexual arousal disorders, orgasmic disorders, and sexual intercourse pain disorders [5]. In China, due to the relatively conservative attitude of Chinese traditional culture toward sex, there is little discussion and research on sexual issues. The research on sexual life and sexual function in patients with diseases mainly focuses on patients with malignant tumors, pregnancy or postpartum, and patients with chronic diseases such as diabetes and hypertension. There are very few studies on the status of sexual life in patients with SLE [6-8]. Among them, a study of the effects of immunosuppressants on sexual function in patients with SLE pointed out that after patients with SLE received immunosuppressant therapy, sexual function indicators of sexual desire, sexual arousal, vaginal lubrication, orgasm, sexual satisfaction, and pain during intercourse changed significantly [6]. Immunosuppressive agents can decrease sex hormone levels and reduce sexual function in patients with SLE. Another study found that the

${ }^{\star}$ Correspondence to: Ying-Hua Pan, Department of Rheumatology, The Third Affiliated Hospital of Sun Yat-sen University, 600 Tianhe Road, Guangzhou 510600, China, E-mail: panyinghua2012@163.com

Dan-Chun Wu, Department of General medicine, The Third Affiliated Hospital of Sun Yat-sen University, 600 Tianhe Road, Guangzhou 510600, China, E-mail: 619165892@qq.com

Key words: systemic lupus erythematosus; female sexual dysfunction; SF-36; FSFI Received: March 24, 2020; Accepted: April 01, 2020; Published: April 06, 2020 
incidence of sexual dysfunction in women with SLE in China was high, and the main types of dysfunction are hyposexuality and difficulty in arousal. The primary influencing factors are body image disturbances [9]. Studies have also shown that sexual function in patients with rheumatic diseases is impaired. The amount of sex enjoyed by patients with rheumatic diseases decreased, and their sexual dysfunction index increased significantly. For example, patients with rheumatic diseases are prone to vaginal dryness, vaginal ulcers, and difficulty in sexual intercourse, and thus, their sexual satisfaction is reduced [10]. People with rheumatic diseases are more likely to experience inferiority and depression, which can lead to decreased sexual desire [11]. The sexual life research of patients with SLE is concentrated primarily in European and North American countries, but rare in China. Studies have found that the pregnancy rate of patients with SLE is significantly lower than that of the general population, mainly due to gonadal damage and decreased quality of sexual life caused by disease treatment [12].

Patients with SLE have a significantly reduced number of sexual episodes, manifested by refusal to have intercourse; and the more active the disease, the greater the sexual function impairment of the patient [6]. SLE patients' disease progression is significantly related to the Female Sexual Function Index (FSFI), sexual desire, sexual arousal, vaginal lubrication, orgasm, and sexual intercourse pain, which may be due to psychological interference caused by the disease $[8,13]$. One of the main reasons is the physical disorder caused by disease treatment. However, a recent meta-analysis showed that there was no significant statistical correlation between SLE disease and sexual desire, sexual arousal, vaginal lubricity, orgasm, sexual satisfaction, and sexual intercourse pain, but it was negatively correlated with the FSFI, which is decreased in women with SLE patients $[5,14]$. This suggests that more research is needed on the status of sexual life in patients with SLE, the interference of the disease on the sexual life of patients, and its influencing factors. In this study, we aimed to determine the effect of SLE on sexual function of female patients in China, using questionnaire survey.

\section{Materials and methods}

\section{Patients}

From April 2018 to May 2019, a total of 204 women with SLE who were treated in the Department of Immunology and Rheumatology at our hospital were investigated for this study. The inclusion criteria were as follows: local residence for more than 5 years; heterosexual; has a regular sexual partner; sexual activity within the last 4 weeks; and has the cognitive ability to understand the content of the questionnaire and cooperate to complete the survey. The exclusion criteria were as follows: pregnant or lactating women; self or partner currently has unstable and serious physical disease that has significant effects on sexual function, such as brain organopathy, cardiovascular disease, endocrine disease, systemic immune disease, or kidney disease; partner has sexual dysfunction; and a history of trauma and surgery that affects sexual life.

\section{General information questionnaire}

The general information questionnaire is designed according to the literature, including age, education, fertility, work status, living conditions, medical payment methods, organ involvement, and whether the patient has facial rashes, mouth ulcers, joint pain, and Raynaud phenomenon.

\section{The 36-item short form health survey (SF-36) and hospital anxiety and depression scale (HADS)}

The Chinese version modified by Li Lu et al. based on the standard SF-36 of the New England Institute of Medical and Health Sciences in Boston, USA, was used. The scale consists of 36 items, which are divided into eight dimensions: physiological function, physiological function, physical pain, overall health, vitality, social function, emotional function, and mental health. Each dimension of the scale has a score ranging from 0 to 100 . The higher the score, the higher the quality of life represented by the dimension. The higher the overall score, the better your health. The HADS contains two items, anxiety and depression. The scores reflect the emotional status of the patients during the treatment.

\section{Female sexual function index (FSFI)}

There are 19 items on the FSFI scale, including sexual desire (items 1-2), sexual arousal (items 3-6), vaginal lubrication (items 7-10), orgasm (items 11-13), sexual life satisfaction (items 14-16), and sexual intercourse pain (items 17-19). The scale has six dimensions, and each dimension scores 2-6 points; the total score is the sum of each item, 36 points. Among them, Dimensions 1 and 2 cover diminished libido or sexual arousal difficulties; Dimension 3 covers vaginal lubrication difficulties; and Dimension 4 covers orgasmic disorder. Dimensions 5 and 6 cover decreased sexual satisfaction or pain during intercourse. An individual is classified as having sexual dysfunction with a total score of $<26.5$. The content validity index of the scale is 0.953 , and the Cronbach a coefficient of each dimension is 0.757 to 0.865 .

\section{Data collection}

All the questionnaires were distributed by the researchers themselves, and the patients were received uniform and clear instructions on how to complete the questionnaire. For patients who could not complete the questionnaire themselves, the researcher verbally went through the questions with the patient. The questionnaire takes approximately 30 minutes for the patient to fill out. A total of 54 questionnaires were distributed. Invalid questionnaires were eliminated. The 52 questionnaires recovered were valid, and the effective recovery rate was $96.3 \%$.

\section{Statistical methods}

We checked the collected survey data, organized it, and used Excel 2010 and SPSS 22.0 software for double entry and analysis. The data are described by frequency and percentage, and measurement data are described by mean \pm standard deviation $(\mathrm{x} \pm \mathrm{SD})$. We analyzed general information, quality of life, and FSFI scores. Multivariate linear regression analysis was performed using general information and quality of life scores as independent variables and FSFI score as the dependent variable. The difference was statistically significant with $\mathrm{P}$ $<0.05$.

\section{Results}

\section{General information of the research object}

A total of 204 women were effectively surveyed. The average age of the survey respondents was $31.67 \pm 1.087$ years, with the largest group in the $20-40$ age group, accounting for $88.4 \%$. The highest education achieved for the majority of them was high school (including technical secondary school), with 51 people, accounting for $25 \%$. The marital 
status was mainly married, with a total of 156 people, accounting for $76.4 \%$. The fertility status was mainly non-fertile, with a total of 115 people, accounting for $56.4 \%$. For working status, 118 women were employed, accounting for $57.8 \%$. For living status, 196 patients are living with their partner accounting for $96.1 \%$. Among the payment methods of medical expenses, medical insurance was the primary method, with a total of 133 , accounting for $65.4 \%$. In terms of income, 74 people had an annual income less than 60,000 , accounting for $36.3 \%$. The complications of SLE disease were as follows: organ involvement (71.2\%), oral ulcers (59.6\%), facial rash (50\%), joint pain (42.3\%), and Raynaud phenomenon (55.8\%).

\section{Quality of life of the study subjects}

The total score of the SF-36 in women with SLE was 108.6 \pm 13.4 , as follows: physiological function was $75.8 \pm 20.2$, physiological function was $27.3 \pm 39.8$, physical pain was $68.2 \pm 18.5$, general health was $57.6 \pm 21.1$, energy was $58.3 \pm 17.5$, social function was $69.6 \pm 22.1$, emotional function was $45.7 \pm 43.8$, and mental health was $69.9 \pm 13.8$.

\section{Status of sexual life and sexual function of the study subjects}

The results of this survey show that the 204 women's FSFI score was $21.4 \pm 8.6$, and sexual desire, sexual arousal, vaginal lubrication, orgasm, sexual satisfaction, and sexual intercourse were scored in six dimensions. They were $2.8 \pm 1.2$ points, $3.12 \pm 1.6$ points, $4.0 \pm 1.9$ points, $3.4 \pm 1.7$ points, $3.9 \pm 1.4$ points, and $4.1 \pm 1.9$ points, respectively.

\section{Correlation of FSFI with general data, SF-36 and HADS scores of women with SLE}

FSFI total score was negative correlated with age, organs involvement and arthralgia. Sexual desire dimension scores were correlated with age, facial rash, arthralgia and raynaud's phenomenon. Sexual arousal dimension scores were correlated with age, organs involvement and arthralgia. Vaginal lubrication dimension scores were correlated with age and fertility status. The score of orgasm dimension is related to age. Sexual satisfaction dimension scores were affected by age and facial rash. Sexual pain dimension scores were influenced by age, organs involvement, arthralgia and raynaud's phenomenon (Table 1).

The correlation between FSFI and SF-36, HADS scores before and after effective treatment was determined. The results showed that the sexual function of female with SLE was associated with SF-36 and HADS scores, including physical functioning, physical role, anxiety and depression dimensions (Table 2). When the patients received effective treatments, their sexual functions were improved greatly. According to our data, vitality and emotional role dimensions in SF-36 turned out significantly associated with the FSFI scores. For HADS, the negative correlations were reversed after the effected treatments (Table 2).

\section{Multiple linear regression analyses of FSFI scores and influencing factors in female SLE patients}

Multivariate linear regression analyses were performed with the total score of FSFI and the score of each dimension as the dependent

Table 1. Association of clinical characteristics of SLE patients and FSFI scores

\begin{tabular}{|c|c|c|c|c|c|c|c|}
\hline Variable & Desire & Arousal & Lubrication & Orgasm & Satisfaction & Pain & TOTAL \\
\hline Age & $-0.384^{a}$ & $-0.298^{a}$ & $-0.402^{a}$ & $-0.325^{a}$ & $-0.245^{a}$ & $-0.224^{a}$ & $-0.378^{a}$ \\
\hline Education & 0.145 & 0.176 & 0.219 & 0.210 & 0.153 & 0.153 & 0.121 \\
\hline Marriage & -0.046 & -0.035 & -0.066 & -0.002 & -0.021 & 0.035 & -0.044 \\
\hline Fertility status & -0.122 & 0.081 & $-0.256^{a}$ & -0.067 & -0.102 & -0.138 & -0.031 \\
\hline Work status & -0.317 & 0.096 & 0.052 & 0.107 & 0.100 & 0.076 & 0.041 \\
\hline Dwelling status & -0.069 & -0.077 & -0.189 & -0.093 & -0.027 & -0.062 & -0.064 \\
\hline Payment method & 0.074 & 0.036 & 0.113 & 0.021 & 0.026 & 0.023 & 0.030 \\
\hline Income/person (yuan) & 0.093 & 0.036 & -0.024 & 0.044 & 0.020 & 0.025 & -0.046 \\
\hline Organs involvement & -0.402 & $-0.243^{a}$ & -0.202 & -0.106 & -0.103 & $0.328^{a}$ & $-0.284^{a}$ \\
\hline Dental ulcer & 0.048 & 0.104 & -0.102 & -0.087 & -0.201 & 0.098 & -0.103 \\
\hline Facial rash & $-0.334^{a}$ & 0.098 & -0.132 & 0.065 & $0.213^{\mathrm{a}}$ & 0.036 & 0.104 \\
\hline Arthralgia & $-0.322^{a}$ & $-0.254^{a}$ & -0.124 & 0.087 & 0.106 & $0.321^{a}$ & $-0.264^{a}$ \\
\hline Raynaud's & $-0.286^{a}$ & -0.086 & 0.101 & 0.098 & 0.074 & $0.304^{a}$ & 0.202 \\
\hline
\end{tabular}

a $P<0.05$. SLE, systemic lupus erythematosus; FSFI, female sexual function index.

Table 2. Association of SF-36, HADS scores in SLE patients and FSFI scores before and after effective treatment

\begin{tabular}{|c|c|c|c|c|c|c|c|c|c|c|c|c|c|c|}
\hline \multirow{2}{*}{ Variable } & \multicolumn{2}{|c|}{ Desire } & \multicolumn{2}{|c|}{ Arousal } & \multicolumn{2}{|c|}{ Lubrication } & \multicolumn{2}{|c|}{ Orgasm } & \multicolumn{2}{|c|}{ Satisfaction } & \multicolumn{2}{|c|}{ Pain } & \multicolumn{2}{|c|}{ TOTAL } \\
\hline & Before & After & Before & After & Before & After & Before & After & Before & After & Before & After & Before & After \\
\hline \multicolumn{15}{|l|}{ SF-36 } \\
\hline Physical functioning & $0.346^{a}$ & $0.487^{a}$ & 0.278 & $0.368^{a}$ & $0.345^{\mathrm{a}}$ & $0.438^{\mathrm{a}}$ & 0.243 & $0.392^{\mathrm{a}}$ & 0.298 & $0.358^{a}$ & $0.312^{\mathrm{a}}$ & 0.182 & $0.394^{a}$ & $0.468^{a}$ \\
\hline Physical role & $0.352^{\mathrm{a}}$ & $0.378^{a}$ & $0.323^{a}$ & $0.346^{a}$ & 0.268 & $0.382^{\mathrm{a}}$ & $0.342^{\mathrm{a}}$ & $0.386^{a}$ & $0.352^{\mathrm{a}}$ & $0.462^{\mathrm{a}}$ & $0.351^{\mathrm{a}}$ & $0.282^{a}$ & $0.343^{\mathrm{a}}$ & $0.423^{a}$ \\
\hline Bodily pain & -0.126 & -0.136 & -0.128 & -0.146 & -0.114 & -0.164 & $-0.309^{a}$ & $-0.346^{a}$ & -0.096 & 0.015 & -0.033 & 0.061 & -0.141 & -0.127 \\
\hline General health & 0.164 & 0.074 & 0.235 & 0.047 & 0.164 & $0.343^{\mathrm{a}}$ & 0.073 & 0.044 & -0.043 & 0.262 & 0.06 & 0.128 & 0.124 & 0.074 \\
\hline Vitality & 0.213 & $0.346^{a}$ & 0.121 & $0.346^{\mathrm{a}}$ & 0.098 & $0.365^{\mathrm{a}}$ & 0.264 & $0.368^{a}$ & 0.192 & $0.402^{\mathrm{a}}$ & 0.224 & $0.422^{a}$ & 0.102 & $0.386^{a}$ \\
\hline Social functioning & 0.122 & 0.128 & 0.121 & 0.245 & 0.023 & 0.126 & 0.123 & 0.089 & 0.104 & 0.123 & 0.121 & 0.123 & 0.122 & 0.143 \\
\hline Emotional role & 0.282 & $0.368^{\mathrm{a}}$ & 0.177 & $0.369^{\mathrm{a}}$ & $0.346^{a}$ & $0.421^{\mathrm{a}}$ & 0.258 & $0.386^{a}$ & $0.325^{\mathrm{a}}$ & $0.464^{a}$ & 0.289 & $0.425^{a}$ & $0.304^{\mathrm{a}}$ & $0.426^{a}$ \\
\hline Mental health & 0.108 & 0.206 & 0.080 & 0.214 & 0.203 & 0.234 & 0.120 & 0.215 & 0.192 & 0.186 & 0.108 & 0.203 & 0.220 & 0.234 \\
\hline \multicolumn{15}{|l|}{ HADS } \\
\hline Total & $-0.394^{a}$ & -0.265 & $-0.321^{a}$ & -0.189 & -0.21 & -0.116 & $-0.303^{\mathrm{a}}$ & -0.132 & $-0.300^{\mathrm{a}}$ & -0.231 & $-0.417^{\mathrm{a}}$ & $-0.315^{a}$ & $-0.416^{a}$ & -0.264 \\
\hline Anxiety & $-0.386^{a}$ & -0.262 & $-0.302^{a}$ & -0.128 & $-0.402^{a}$ & -0.245 & $-0.313^{a}$ & -0.130 & $-0.316^{a}$ & -0.215 & $-0.325^{a}$ & -0.249 & $-0.308^{a}$ & -0.221 \\
\hline Depression & $-0.361^{a}$ & -0.113 & $-0.401^{a}$ & $-0.322^{a}$ & $-0.317^{a}$ & -0.238 & $-0.322^{a}$ & -0.126 & $-0.321^{a}$ & -0.124 & $-0.360^{a}$ & $-0.331^{a}$ & $-0.324^{a}$ & -0.272 \\
\hline
\end{tabular}

${ }^{a} P<0.05$. SLE, systemic lupus erythematosus; FSFI, female sexual function index; HADS, hospital anxiety and depression scale. 
variable, and the corresponding index with statistical significance in the correlation analysis as the independent variable (Supplementary Tables 1-7). The results showed that: education level was the influencing factor of the FSFI sexual arousal, orgasm, and sexual desire dimensions; medical payment method was the influencing factor of sexual arousal, orgasm, desire, and intercourse pain dimensions; facial rash affects sexual arousal dimension; marital status, reproductive status, and work status are factors influencing orgasm, sexual desire, and intercourse pain dimensions; the emotional role of SF-36 and Raynaud phenomenon are factors influencing the intercourse pain and sexual desire dimensions; energy, the total score of SF-36, and organ involvement are factors influencing the sexual intercourse pain dimension; bodily pain and emotional function of SF-36, and joint pain, affect the sexual desire dimension.

\section{Discussion}

Sex life and sexual function, as the basic components of quality of life, have special significance for patients with SLE [15]. Due to the impact of the disease and treatment, difficulty with sex life and sexual function run through all aspects of these patients' physical, psychological, and social lives. It should be noted that the sex life and sexual function of patients are also closely related to the health education provided by medical personnel on the sexual aspects of living with the disease [16]. Some patients with chronic diseases believe that medical staff should guide their sexual life after treatment. Studies have shown that medical staff can provide consultation and intervention measures for patients after treatment to help improve patients' quality of life and sexual function. In the context of China's special culture, exploring the sexual function of patients with SLE and its influencing factors will help medical personnel recognize the importance of the problem and take measures to improve the quality of the sexual life of patients.

\section{Women with SLE and FSD deserve attention}

The sex hormone estradiol is a natural estrogen that promotes endometrial hyperplasia, enhances the contraction of uterine smooth muscle, and has an anti-male effect, which is related to the experience and quality of a woman's sexual life. Ovarian function in patients with immunosuppressants decreased, resulting in a decrease in estradiol levels in patients with SLE [17]. Active drugs such as cyclophosphamide inhibit the function of the ovaries by combining with enzymes in the DNA cells, and cause the number of primary follicles and stimulated follicles to be significantly reduced, resulting in a decrease in ovarian function. After application of immunosuppressants, there are significant differences in sexual desire, sexual arousal, vaginal lubrication, orgasm, sexual satisfaction, and pain during intercourse, which indicates that immunosuppressants have a suppressive effect on sexual function, for which the reasons may be analyzed and the levels of sex hormones may be reduced. In Asian culture, sex culture is more traditional and conservative, and sexual issues are rarely discussed openly. Previous studies have shown that the incidence of FSD in different ethnic groups is different due to different cultural backgrounds [18].

\section{Influencing factors of FSD in women with SLE}

Lower education may be a common feature of the predisposed population of FSD. A study of women with autoimmune diseases showed that factors such as patient age, education, occupation, monthly income, and course of disease are factors influencing FSD, and that disease activity index and pain caused by disease are also related to FSD $[13,14]$. In this study, education, living conditions, and medical expenses did not affect the sexual function of female SLE patients. Involved organs, oral ulcers, facial rash, joint pain, and Raynaud phenomenon were related to FSFI. The scores of physiological function, physical pain, general health, energy, social function, emotional function, and mental health in SF-36 are correlated with the FSFI score of women with SLE. Patients with require long courses of glucocorticoids and immunosuppressants, which cause increased fatigue, and they often lose time from their job due to recurrent illness, in turn resulting in anxiety and depression. The medical cost of this disease is also one of the influencing factors.

Different social backgrounds and cultural differences of patients will lead to different rates of FSD in women with SLE. The results of influencing factor analyses are not completely consistent across regions. Based on this, multiple linear regression analysis was performed on the factors related to the occurrence of FSD. The results showed that education level was an influencing factor of FSFI scores of sexual arousal dimension, orgasm dimension, and sexual desire dimension. Marital status and fertility status are the influencing factors of sexuality score. Medical expenses payment method is an influencing factor of the FSFI orgasm dimension score in women with SLE. Energy, emotional function, SF-36 total score, work status, living status, medical expenses payment method, involvement of organs, and Raynaud phenomenon are influencing factors of the pain sexual dimension score.

\section{Significance and limitations of the study}

Women's status in today's society is constantly improving, and the quality of sexual life of female patients has also received more attention. The results of this study show that women with SLE often have sexual dysfunction that can have serious impacts on society and families. It is recommended that we formulate corresponding measures for the main influencing factors of FSD in women with SLE in clinical practice and do a good job of evaluating and intervening in the quality of sexual life of this population. These steps will improve the quality of life of patients with SLE. Although this study strictly examined the qualifications of the investigators and tried to specifically study the relevant factors affecting sexual function, no general data and psychological factors were stratified, and no control group was established. The sample size has certain limitations. It should be noted that the sexual life and sexual function of patients are also closely related to the health education of medical personnel on sexual aspects. Some patients with chronic diseases would welcome information to guide their sexual life. Some studies have shown that medical staff can provide patients with counseling and intervention after treatment to help improve the quality of life and sexual function of patients.

\section{Conflict of interest}

The authors declare no conflict of interest.

\section{Acknowledgement}

This study is supported by Nursing Research Foundation of Guangdong Nursing Association (gdhlxueh2019zx 099).

\section{References}

1. Nusbaum JS, Mirza I, Shum J, Freilich RW, Cohen RE, et al. (2020) Sex differences in systemic lupus erythematosus: epidemiology, clinical considerations, and disease pathogenesis. Mayo Clin Proc 95: 384-394. [Crossref]

2. Aringer M (2019) Inflammatory markers in systemic lupus erythematosus. J Autoimmun 1: 102374 .

3. Zhang CX, Cai L, Zhou ZY, Mao YY, Huang H, et al. (2019) Clinical manifestations, immunological features and prognosis of chinese pediatric systemic lupus erythematosus: A single-center study. Int J Rheum Dis 22: 1070-1076. 
4. Raja TW, Duraipandiyan V, Ignacimuthu S, Al-Dhabi NA (2020) Current trends in the treatment of systemic lupus erythematosus. Curr Pharm Des 1: 1-10. [Crossref]

5. Clayton AH, Valladares Juarez EM (2019) Female sexual dysfunction. Med Clin North Am 103: 681-698

6. Moghadam ZB, Rezaei E, Faezi ST, Zareian A, Ibrahim FM, et al. (2019) Prevalence of sexual dysfunction in women with systemic lupus erythematosus and its related factors. Reumatologia 57: 19-26. [Crossref]

7. Patoulias DI (2019) Sexual dysfunction among women with systemic lupus erythematosus: might it reflect cardiovascular disease burden? Reumatologia 57: 120123. [Crossref]

8. Godoy Junior AC, Gaertner H, Skare T, Nisihara R (2017) Sexual dysfunction in systemic lupus erythematosus patients. Acta Reumatol Port 42: 341-342

9. Shen B, He Y, Chen H, Zhao C, Zhu L, et al. (2015) Body image disturbances have impact on the sexual problems in chinese systemic lupus erythematosus patients. $J$ Immunol Res 2: 204513. [Crossref]

10. Favalli EG, Biggioggero M, Crotti C, Becciolini A, Raimondo MG, et al. (2019) Sex and management of rheumatoid arthritis. Clin Rev Allergy Immunol 56: 333-345.

11. Ostensen M (2017) Sexual and reproductive health in rheumatic disease. Nat Rev Rheumatol 13: 485-493.
12. Pastore DEA, Costa ML, Surita FG (2019) Systemic lupus erythematosus and pregnancy: the challenge of improving antenatal care and outcomes. Lupus 28: 14171426. [Crossref]

13. Garcia Morales M, Callejas Rubio JI, Peralta-Ramirez MI, Henares Romero LJ, Rios Fernandez R, et al. (2013) Impaired sexual function in women with systemic lupus erythematosus: a cross-sectional study. Lupus 22: 987-995.

14. Yin R, Xu B, Li L, Fu T, Zhang L, et al. (2017) The impact of systemic lupus erythematosus on women's sexual functioning: A systematic review and meta-analysis. Medicine (Baltimore) 96: e7162. [Crossref]

15. Olesinska M, Saletra A (2018) Quality of life in systemic lupus erythematosus and its measurement. Reumatologia 56: 45-54.

16. Phuti A, Schneider M, Makan K, Tikly M, Hodkinson B (2019) Living with systemic lupus erythematosus in South Africa: a bitter pill to swallow. Health Qual Life Outcomes 17: 60-65.

17. Ward JM, Rider V, Abdou NI, Kimler B (2013) Estradiol differentially regulates calreticulin: a potential link with abnormal $\mathrm{T}$ cell function in systemic lupus erythematosus? Lupus 22: 583-596. [Crossref]

18. Avis NE, Zhao X, Johannes CB, Ory M, Brockwell S, et al. (2018) Correlates of sexua function among multi-ethnic middle-aged women: results from the study of women's health across the nation (SWAN). Menopause 25: 1244-1255. [Crossref]

Copyright: (C2020 Pan Y. This is an open-access article distributed under the terms of the Creative Commons Attribution License, which permits unrestricted use, distribution, and reproduction in any medium, provided the original author and source are credited. 